Kanso E., Arroyo M., Tong Y., Yavari A., Marsden J.E. and Desbrun M, On the geometric character of stress in continuum mechanics, Zeitschrift für

Angewandte Mathematik und Physik (ZAMP), Vol. 58, Issue 5, pp. 843-856, 2007

\title{
On the geometric character of stress in continuum mechanics
}

Eva Kanso, Marino Arroyo, Yiying Tong, Arash Yavari, Jerrold E Marsden ${ }^{1}$ and Mathieu Desbrun

\begin{abstract}
This paper shows that the stress field in the classical theory of continuum mechanics may be taken to be a covector-valued differential two-form. The balance laws and other fundamental laws of continuum mechanics may be neatly rewritten in terms of this geometric stress. A geometrically attractive and covariant derivation of the balance laws from the principle of energy balance in terms of this stress is presented.
\end{abstract}

Mathematics Subject Classification (2000).

Keywords. Continuum mechanics, elasticity, stress tensor, differential forms.

\section{Motivation}

This paper proposes a reformulation of classical continuum mechanics in terms of bundle-valued exterior forms. Our motivation is to provide a geometric description of force in continuum mechanics, which leads to an elegant geometric theory and, at the same time, may enable the development of space-time integration algorithms that respect the underlying geometric structure at the discrete level.

In classical mechanics the traditional approach is to define all the kinematic and kinetic quantities using vector and tensor fields. For example, velocity and traction are both viewed as vector fields and power is defined as their inner product, which is induced from an appropriately defined Riemannian metric. On the other hand, it has long been appreciated in geometric mechanics that force should not be viewed as a vector, but rather a one-form. This fits naturally with one of the main properties of a force, namely that when paired with a displacement (a vector), one gets work. No metric is needed for this operation of course when force is thought of as a one form. One also sees the same thing when one looks at the tensorial nature of the Euler-Lagrange equations: the equations themselves are natually one-form equations, not vector equations. Despite this, the notion of force as a one-form has not properly been put into the foundations of continuum mechanics. In the

\footnotetext{
${ }^{0}{ }^{1}$ Research partially supported by the California Institute of Technology and NSF-ITR Grant ACI-0204932.
} 
geometric approach to continuum mechanics proposed in this paper, traction is defined as an exterior one-form. Consequently, one also has a metric-independent notion of power as the natural pairing between the velocity vector field and the traction one-form.

Although the importance of the geometric character of these fields is already known in mechanics (see, for example, [17] and [3]), the classical derivation of the balance laws as presented in most works does not reflect this geometric understanding. One of the purposes of the present work is to fill this gap.

An outcome of this approach is that the stress field is naturally described as a bundle-valued two-form. The balance laws are then rewritten in terms of the new geometric stress by appealing to tools from differential calculus on bundlevalued forms; that is, in terms of Cartan's calculus [2]. It is worth noting that the notion of stress as a covector-valued two-form appears in the recent literature, e.g., $[18,9,13,6]$, but a reformulation of the balance laws in terms of this stress in arbitrary Riemannian ambient spaces has remained open. This paper fills that gap and provides a complete treatment of continuum mechanics, including balance laws and constitutive equations, in terms of this geometric notion of the stress.

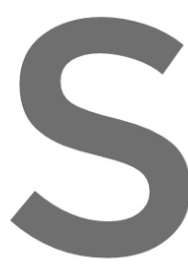

The reformulation of elasticity in terms of (bundle-valued) exterior forms bring e theory closer to Discrete Exterior Calculus (DEC), see, e.g., [14] and [5], and,
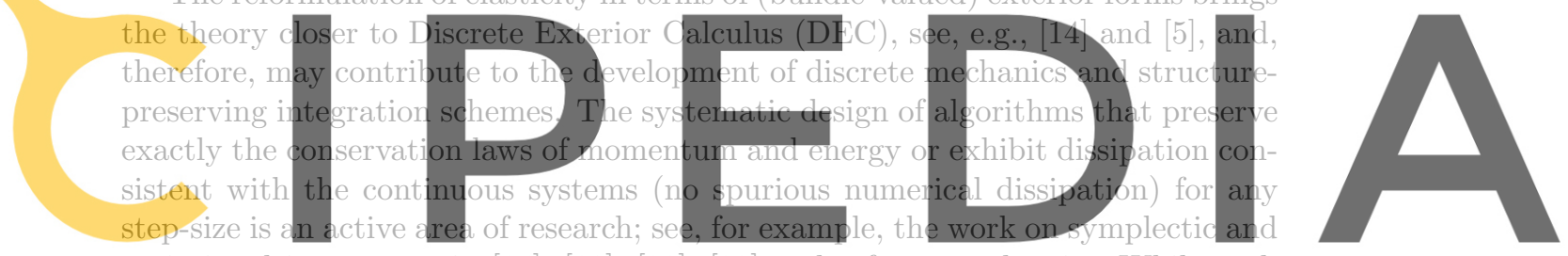

Register for free at htthe integators for finitedimensional mechanical swstems are well-understood, the watermark

ical quantities (such as stress and strain) and the symmetries of the equations (such

as conservation of momentum and energy) remain a challenge. Discrete Exterior

Calculus alone might not, in itself, be sufficient for the design of such conserving algorithms but may provide some useful tools for this undertaking. We view the present study, that is, the geometric reformulation of elasticity, as a first step in our research project on developing a consistent theory of discrete elasticity that will lead to the design of geometric space-time integration algorithms.

The organization of this paper is as follows: In $\S 2$ we introduce the stress as a bundle-valued form and rewrite the classical balance laws and constitutive relations in terms of this geometric stress. In $\S 3$, we assume the existence of a stress form, with no reference to the stress tensor, and present a covariant derivation of the balance laws and constitutive equations. The results are summarized in $\S 4$. 


\section{Classical continuum mechanics in terms of bundle-valued forms}

Bodies and motions. As in traditional continuum mechanics, a body is a set of particles, or material points, which are often regarded as a subset of Euclidean 3 -space. Following the geometric view of continuum mechanics ${ }^{1}$, in this paper we shall regard a body as a Riemannian manifold $\mathcal{R}_{0}$ with boundary, whose metric tensor is denoted G. Of course a standard example would be an open set with a sufficiently smooth boundary in Euclidean 3-space $\mathbb{R}^{3}$ for three-dimensional elasticity and a domain in $\mathbb{R}^{2}$ for shells, each with the standard metric. The body is assumed to deform in an ambient Riemannian space $\mathcal{S}$ with metric $\mathrm{g}$ (Fuclidean 3-space in the standard examples). A deformation is a map $\varphi: \mathcal{R}_{0} \rightarrow \mathcal{S}$ that is a diffeomorphism from $\mathcal{R}_{0}$ onto its image, the deformed body $\mathcal{R}=\varphi\left(\mathcal{R}_{0}\right) \subset \mathcal{S}$. The deformed body inherits the Riemannian structure of $\mathcal{S}$; see Figure 1. A motion is a curve of deformations, that is, a one-parameter (time dependent) family of deformation maps and may be represented as

$$
\mathrm{X}=\varphi_{t}(\mathbf{X})=\varphi(\mathbf{X}, t),
$$

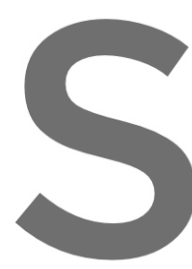

where $\mathrm{X}$ and $\mathrm{x}$ denote, respectively, the position of a material particle in the fixed reference configuration $\mathcal{R}_{0}$ and $\mathbf{k}$ s position in the current configuration $\mathcal{R}$.
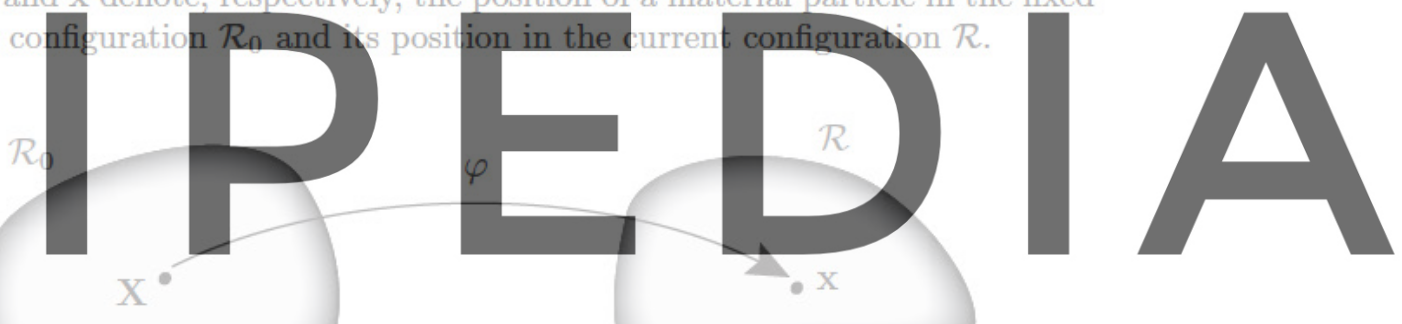

Register for free at https//www.scipedia.com to download the version without the watermark

Figure 1. The deformation map $\varphi$ takes the reference configuration $\mathcal{R}_{0}$ to the current configuration $\mathcal{R}$.

Further background and notation. The tangent bundle of $\mathcal{R}$ is denoted by $T \mathcal{R}$ and the cotangent bundle by $T^{*} \mathcal{R}$. Elements of $T \mathcal{R}$ are called vectors and those of $T^{*} \mathcal{R}$ are covectors or one-forms. Consider a coordinate basis $\mathbf{e}_{i}=\partial / \partial x^{i}$, $i=1,2,3$ for $T \mathcal{R}$ and its dual basis $\mathrm{e}^{i}=\mathrm{d} x^{i}$ for $T^{*} \mathcal{R}$, where $x^{i}$ represents the local coordinates of a point $\mathrm{x}$ in $\mathcal{R}$. Recall that a covariant, second-order tensor field (or 2-tensor) $\mathbf{T}$ on $\mathcal{R}$ is a bilinear mapping $\mathbf{T}: T \mathcal{R} \times T \mathcal{R} \rightarrow \mathbb{R}$ and can be expressed in coordinates as $\mathbf{T}=T_{i j} \mathbf{e}^{i} \otimes \mathbf{e}^{j}$, where $\otimes$ denotes the tensor product. A contravariant 2-tensor $\mathbf{S}$ is a bilinear mapping $\mathbf{S}: T^{*} \mathcal{R} \times T^{*} \mathcal{R} \rightarrow \mathbb{R}$, that can be expressed as $\mathbf{S}=S^{i j} \mathbf{e}_{i} \otimes \mathbf{e}_{j}$. One can also define mixed 2-tensors as bilinear

\footnotetext{
${ }^{1}$ It is assumed that the reader is familiar with the geometric approach to continuum mechanics. Further background and details on the notation can be found in [1] and [17] as well as in the research literature, such as [23].
} 
E. Kanso et al.

mappings $T \mathcal{R} \times T^{*} \mathcal{R} \rightarrow \mathbb{R}$ or $T^{*} \mathcal{R} \times T \mathcal{R} \rightarrow \mathbb{R}$. We denote the space of covariant 2-tensor fields on $\mathcal{R}$ by $T_{2}^{0}(\mathcal{R})$, the space of contravariant 2-tensors by $T_{0}^{2}(\mathcal{R})$ and mixed tensors by $T_{1}^{1}(\mathcal{R})$ or $T_{1}^{1}(\mathcal{R})$. This notation extends in the obvious way to $k$-tensors. Further, let $\Omega^{k}(\mathcal{R})$ denote the space of $k$-forms, or alternating $k$-tensors, on $\mathcal{R}$. In particular, $\Omega^{0}(\mathcal{R})$ is the space of smooth functions on $\mathcal{R}$ and $\Omega^{1}(\mathcal{R})$ is the space of smooth sections of $T^{*} \mathcal{R}$. Similarly, we introduce a basis $\mathbf{E}_{i}$ on $T \mathcal{R}_{0}$ and its dual $\mathbf{E}^{i}$ on $T^{*} \mathcal{R}_{0}$ and adopt analogous notation for tensors on $\mathcal{R}_{0}$ as well as for two-point tensors, that is, tensors that can have "legs" in both $\mathcal{R}$ and $\mathcal{R}_{0}$ connected through the diffeormorphism $\varphi: \mathcal{R}_{0} \rightarrow \mathcal{R}$. For example, $T_{1,1}^{0,0}\left(\mathcal{R}, \mathcal{R}_{0}\right)$ denotes the snace of two-point 2-tensors or bilinear mans $T \mathcal{R} \times T \mathcal{R}_{0} \rightarrow \mathbb{R}$.

Finally, the flat $(\cdot)^{b}$ and sharp $(\cdot)^{\sharp}$ operations refer to lowering and raising tensor indices. On $\mathcal{R}$ this would mean using the metric g. For example, ${ }^{b}: T \mathcal{R} \rightarrow T^{*} \mathcal{R}$ is defined by $\left\langle v^{b}, w\right\rangle=\mathrm{g}(v, w)$ and its inverse is ${ }^{\sharp}: T^{*} \mathcal{R} \rightarrow T \mathcal{R}$. Similar operations are defined on the reference configuration $\mathcal{R}_{0}$ with respect to a metric $\mathrm{G}$. Here, the symbol $\langle\cdot, \cdot\rangle$ is used to denote the natural pairing of a contravariant field with a covariant field, such as the pairing of a vector field and a one-form or covector. We shall sometimes use the notation $\langle\langle\cdot, \cdot\rangle\rangle$ to denote the inner product between two covariant or contravariant fields with respect to the corresponding metric

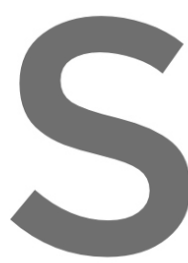
Continuun Mechanics. The body forces per วR. Contin the motion forces are o force b are 1 at
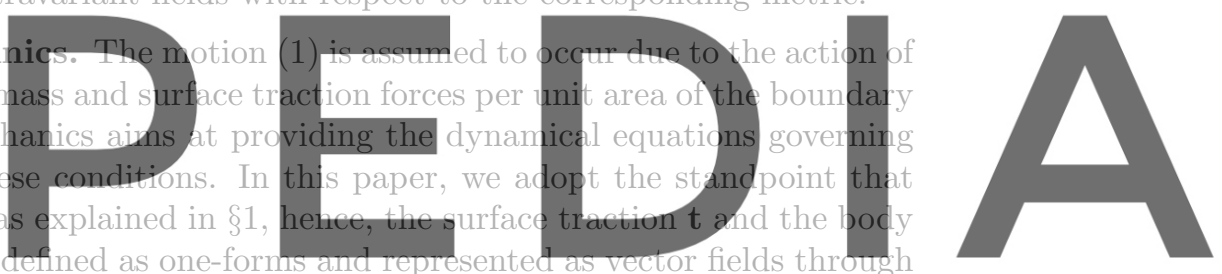

the $\sharp$ operator. This view is important to the development of Elasticity in terms

\subsection{The stress field as a covector-valued two-form}

Surface traction and Cauchy's stress. In the classical non-relativistic theory, the basic postulate for formulating the dynamical equations of motion is the existence of a stress field $\mathbf{t}^{\sharp}(\mathbf{x}, t ; \mathbf{n})$ defined everywhere in $\mathcal{R}$. Physically, $\mathbf{t}^{\sharp}(\mathbf{x}, t ; \mathbf{n})$ represents the force per unit area exerted on a surface element $\mathrm{d} a$ in $\mathcal{R}$ oriented with unit normal $\mathbf{n}$. It is also convenient to introduce the stress field $\mathbf{p}^{\sharp}(\mathbf{X}, t ; \mathbf{N})$ acting on surface elements in $\mathcal{R}$ but measured per unit area of the corresponding surface elements in $\mathcal{R}_{0}$, that is,

$$
\mathbf{p}^{\sharp}(\mathbf{X}, t ; \mathbf{N}) \mathrm{d} A=\mathbf{t}^{\sharp}(\mathbf{x}(\mathbf{X}, t), t ; \mathbf{n}) \mathrm{d} a,
$$

where $\mathrm{d} A$ is an oriented surface element in $\mathcal{R}_{0}$ with unit normal $\mathbf{N}$ and $\mathbf{N d} A$ is related to $\mathbf{n d} a$ by the Piola formula: $\mathbf{n} \mathrm{d} a=J \mathbf{F}^{-\mathrm{T}} \mathbf{N} \mathrm{d} A$. Here, the deformation gradient $^{2}$ is denoted $\mathbf{F}=\partial \varphi / \partial \mathbf{X}\left(=F_{A}^{a} \mathbf{e}_{a} \otimes \mathbf{E}^{A}\right)$ is a mixed 2-tensor $\in T_{0,1}^{1,0}\left(\mathcal{R}, \mathcal{R}_{0}\right)$,

\footnotetext{
2 We cannot resist making the standard remark that despite its misleading name, $\mathbf{F}$ is not a
} 
$J=\operatorname{det}(\mathbf{F}) \sqrt{\operatorname{det}(\mathbf{g}) / \operatorname{det}(\mathbf{G})}$ (det is the determinant), and $(\cdot)^{\top}$ denotes the transpose.

Cauchy's stress theorem states that there are second-order stress tensors called, respectively, the Cauchy stress tensor $\boldsymbol{\sigma}$ and the two-point Piola-Kirchhoff stress tensor $\mathbf{P}$, such that

$$
\mathbf{t}(\mathbf{x}, t ; \mathbf{n})=\langle\boldsymbol{\sigma}(\mathbf{x}, t), \mathbf{n}\rangle, \quad \mathbf{p}(\mathbf{X}, t ; \mathbf{N})=\langle\mathbf{P}(\mathbf{X}, t), \mathbf{N}\rangle .
$$

This means that $\mathbf{t}(\mathbf{x}, t ; \mathbf{n})$ and $\mathbf{p}(\mathbf{X}, t ; \mathbf{N})$ depend linearly on $\mathbf{n}$ and $\mathbf{N}$, respectively.

Rewriting the stress as a covector-valued two-form. Although the physical interpretation of the notion of stress is geometric, their vectorial and tensorial representations fail to exploit, or even reveal, their geometric character. One of the main goals of the present work is to clarify this geometric nature by rewriting the stress fields as covector-valued two-forms.

We will take an approach to stress that considers them to be covector valued two-forms and regards them as fundamental quantities in a manner similar to the way one postulates the existence of $\mathbf{t}(\mathbf{x}, t ; \mathbf{n})$ in the standard approach. However, before taking this point of view, we show how they will end up being related to

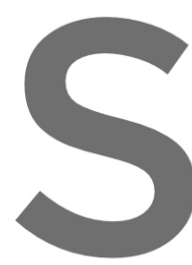

the standares
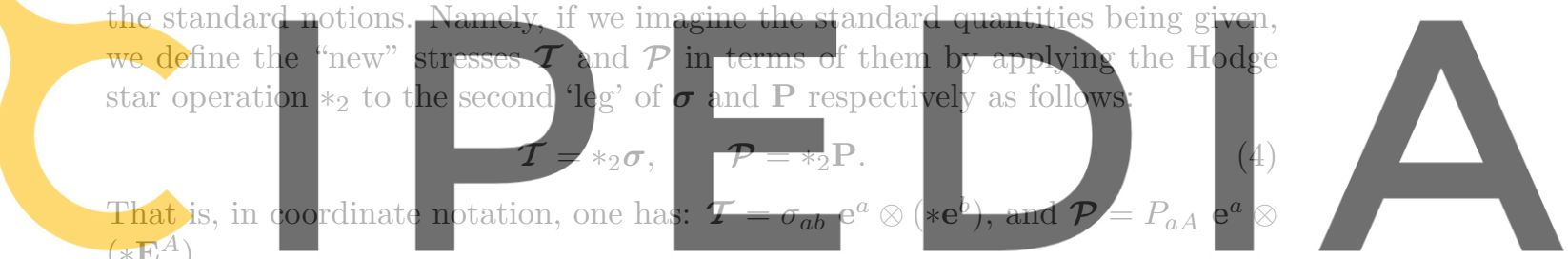

By definition, one obtains $\mathcal{T} \in \Omega^{1}(\mathcal{R}) \otimes \Omega^{2}(\mathcal{R})$ and $\mathcal{P} \in \Omega^{1}(\mathcal{R}) \otimes \Omega^{2}\left(\mathcal{R}_{0}\right) .{ }^{3}$

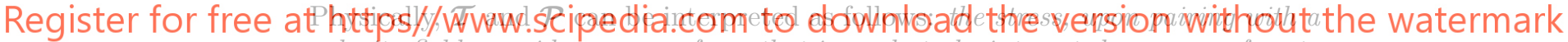

velocity field, provides an area-form that is ready to be integrated over a surface to give the rate of work done by the stress on that surface - this point is elaborated further in $\S 2.2$.

Another point is worth mentioning. That is, part of the linearity of $\mathbf{t}$ is that it switches sign under a change of sign of $\mathbf{n}$. This property is nicely built into the new tensors $\mathcal{T}$ and $\mathcal{P}$ simply because they are two forms - changing the arguments as two-forms switches their signs and this may be regarded as a reflection of the change of orientation of the surface to which $\mathbf{n}$ is normal. However, note that when we say $\mathcal{T}$ and $\mathcal{P}$ are covector valued two forms, there is no need to mention $\mathbf{n}$ or a surface element $\mathrm{d} a$ as such - unless one wants to reconstitute the classical stresses from them using oriented surface elements.

The Piola transformation. Recall that the standard stress tensors $\boldsymbol{\sigma}$ and $\mathbf{P}$ are related through the Piola transformation:

$$
J \boldsymbol{\sigma}=\mathbf{P F}^{T} .
$$

gradient at all, but simply is the derivative of the map $\varphi$.

${ }^{3}$ Or, $\mathcal{T} \in T \mathcal{R} \otimes \Omega^{2}(\mathcal{R})$ and $\mathcal{P} \in T \mathcal{R} \otimes \Omega^{2}\left(\mathcal{R}_{0}\right)$, depending on the representation of $\boldsymbol{\sigma}$ and $\mathbf{P}$. 
Clearly, $\mathbf{P}$ is not the pull-back of $\boldsymbol{\sigma}$ by the motion $\varphi$. But this equation, when written in terms of the stress-forms $\mathcal{T}$ and $\mathcal{P}$, reads as:

$$
\mathcal{P}=\varphi^{*_{2}} \mathcal{T}
$$

where $\varphi^{*_{2}}$ is defined as the pull back by the mapping $\varphi$ of the area-form of a covector-valued two-form, e.g., $\mathcal{T} \in T^{*}(\mathcal{R}) \times \Omega^{2}(\mathcal{R})$, or of the second 'leg' of a two-tensor, e.g., $\boldsymbol{\sigma} \in T^{*}(\mathcal{R}) \times T^{*}(\mathcal{R})$. That is, the Piola transformation in (6) has a clear geometric interpretation: $\mathcal{P}$ is the pull-back of the area-form part of $\mathcal{T}$ that does nothing to the covector-valued part.

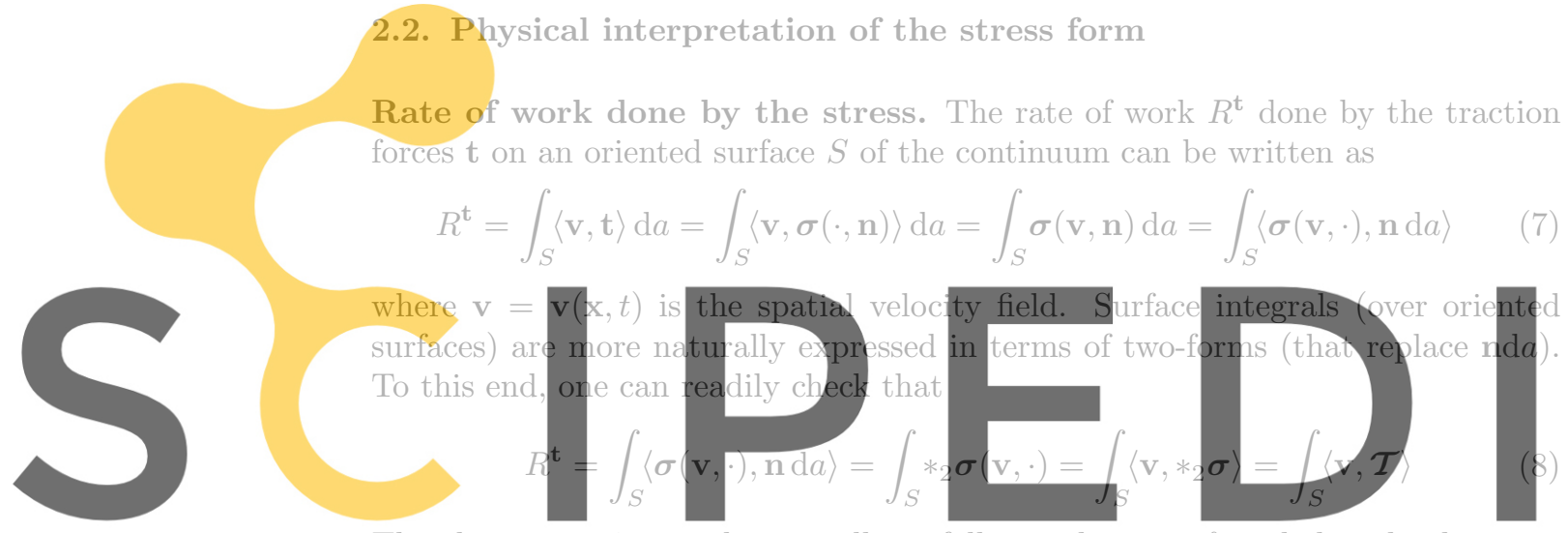

The above equation reads naturally as follows: the rate of work done by the stress

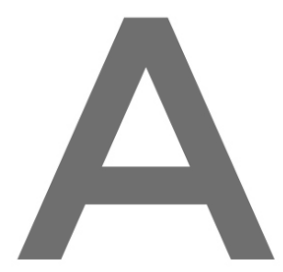

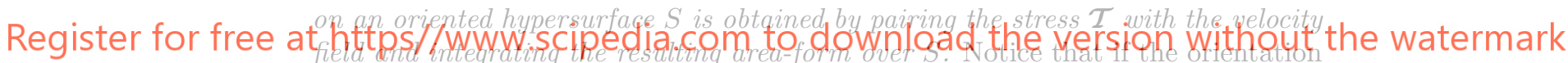

of $S$ switches, then the sign of the integral automatically switches and this corresponds to the change of sign of $n$ in the traditional approach. Similar relations hold for $\mathcal{P}$ and $\mathbf{p}$, namely, $R^{\mathbf{t}}=\int_{S_{0}}\langle\mathbf{V}, \mathcal{P}\rangle$, where $\mathbf{V}$ is the material velocity field defined by $\mathbf{V}(\mathbf{X}, t)=\partial \varphi(\mathbf{X}, t) / \partial t$ and $\mathbf{v}(\mathbf{x}, t)=\mathbf{V}(\mathbf{X}(\mathbf{x}, t), t)$.

The resultant force in Euclidean space. Let the body deform in a Euclidean space. The notion of a resultant force acting on a surface $S$ with unit normal $\mathbf{n}$ depends on the Euclidean structure of the ambient space and is given by $\mathbf{f}=$ $\int_{S} \mathbf{t} \mathrm{d} a=\int_{S}\langle\boldsymbol{\sigma}, \mathbf{n d} a\rangle$. This force can be rewritten as

$$
\mathbf{f}=\int_{S} \mathcal{T}=\int_{S} *_{2} \boldsymbol{\sigma}=\int_{S} \mathbf{e}^{a} \otimes *\left(\sigma_{a b} \mathbf{e}^{b}\right)=\int_{S} \mathbf{e}^{a} \otimes * \mathbf{t}_{a}=\mathbf{e}^{a} \otimes \int_{S} * \mathbf{t}_{a},
$$

where the Euclidean structure allows us to "factor out" the basis vectors of the traction field and integrate the area-form component-wise. The force in the latter expression does not explicitly depend on the normal nda. Also, it is clear that $\mathcal{T}$ automatically obeys Cauchy's lemma in the sense that the resultant force changes sign if we change the orientation of $S$, as we have mentioned previously. 


\subsection{Differentation of bundle-valued forms}

In this section, we define an operation that will be of importance for rewriting the balance laws in terms of $\mathcal{T}$ and $\mathcal{P}$ in $\S 2.4$, namely, a differentiation operation $\mathfrak{d}$ of vector- and covector-valued forms. The differentiation $\mathfrak{d}$ combines the exterior derivative $\mathbf{d}$, that has a topological character, with the covariant derivative $\nabla$ with respect to the Riemannian connection, that has a metric character, see, e.g., [1] and [8]. To this end, recall that, in component notation, the covariant derivative $\nabla \boldsymbol{v}$ of a vector field $\boldsymbol{v}=v^{i} \mathbf{e}_{i}$ on $T \mathcal{R}$ is given by $\nabla_{j} v^{i}=v^{i}{ }_{j j}=\partial v^{i} / \partial x^{j}+\gamma_{j k}^{i} v^{k}$, where $\gamma_{j k}^{i}$ are the Christoffel symbols, also called the connection coefficients. This suggests that $\nabla v$ can be expressed as a mixed 2-tensor, that is, a vector-valued one-form $\nabla \boldsymbol{v}=v^{i}{ }_{\mid j} \mathbf{e}_{i} \otimes \mathbf{e}^{j}$. In particular, one has $\nabla \mathbf{e}_{j}=\mathbf{e}_{i} \otimes \gamma_{j k}^{i} \mathbf{e}^{k}=\mathbf{e}_{i} \otimes \omega_{j}^{i}$, where $\omega_{j}^{i}=\gamma_{j k}^{i} \mathbf{e}^{k}$ are called the connection one-forms.

The derivative $\mathfrak{d}$. Let $\mathbb{T}$ denote either $T \mathcal{R}$ or $T^{*} \mathcal{R}$, and let $k$ be any integer $\leq 3$. We define the differential operator

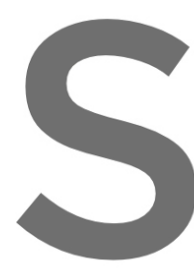

$$
\mathfrak{d}: \mathbb{T} \otimes \Omega^{k-1}(\mathcal{R}) \longrightarrow \mathbb{T} \otimes \Omega^{k}(\mathcal{R}) ; \quad \mathcal{T} \longmapsto \mathfrak{d} \mathcal{T}
$$
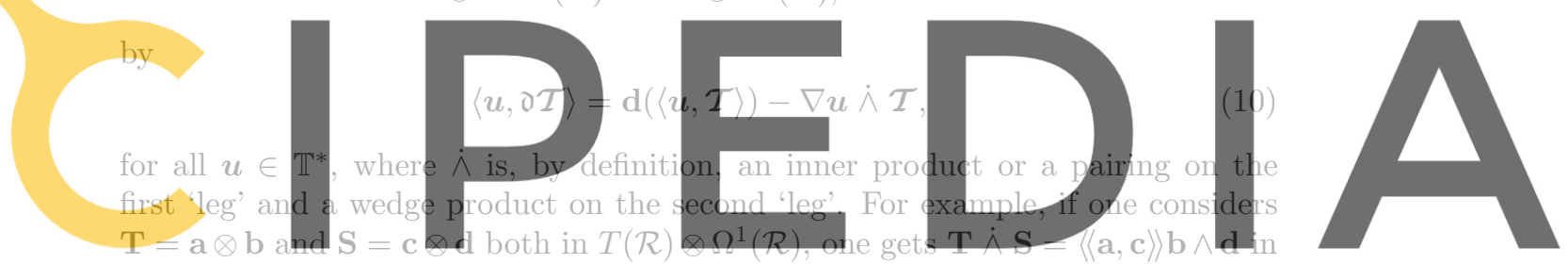

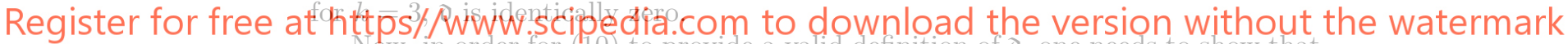
Now, in order for (10) to provide a valid definition of o, one needs to show that

its right hand side depends only on the point values of $u$ and, hence, uniquely defines the differential $\mathfrak{d} \mathcal{I}$. To this end, note that for any function $f \in \Omega^{0}(\mathcal{R})$, one has

$$
\mathbf{d}(\langle f \boldsymbol{u}, \mathcal{T}\rangle)=\mathbf{d}(f \wedge\langle\boldsymbol{u}, \mathcal{T}\rangle)=(\mathbf{d} f) \wedge\langle\boldsymbol{u}, \mathcal{T}\rangle+f \mathbf{d}(\langle\boldsymbol{u}, \mathcal{T}\rangle)
$$

On the other hand, one can readily verify that

$$
\nabla(f \boldsymbol{u}) \dot{\wedge} \mathcal{T}=(\boldsymbol{u} \otimes \mathbf{d} f) \dot{\wedge} \mathcal{T}+f \nabla \boldsymbol{u} \dot{\wedge} \mathcal{T}=(\mathbf{d} f) \wedge\langle\boldsymbol{u}, \mathcal{T}\rangle+f \nabla \boldsymbol{u} \dot{\wedge} \mathcal{T}
$$

which proves our claim. Note that the differential operator $\mathfrak{d}$ is closely related to Cartan's exterior covariant differential (reviewed in [9, Chapter 9], see also [21] and [16]), which was originally developed to express connections and curvatures in terms of forms.

Motivated by the fact that the usual pull-back of forms commutes with the exterior derivative, we are able to use $\mathfrak{d}$ to define a derivative $\mathfrak{D}$ on elements of

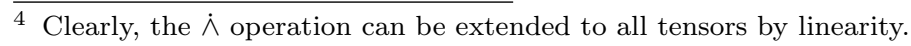


the space $\mathbb{T} \otimes \Omega^{k-1}\left(\mathcal{R}_{0}\right)(k \leq 3)$ such that the following diagram commutes

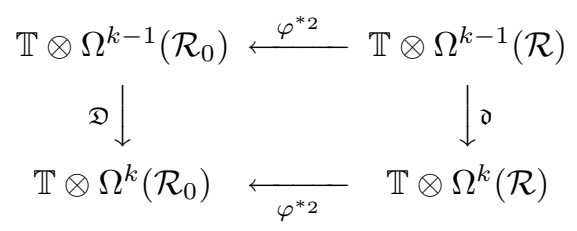

where $\varphi^{*_{2}}$ denotes a partial pullback of the " $(k-1)$-form" part of the tensors but does nothing to the vector or covector values. This diagram simply means that for $\nu \in \mathbb{T}, \omega \in \Omega^{k-1}(\mathcal{R})$, we have

$$
\mathfrak{d}(\nu \otimes \omega)\left(u, \varphi_{*} V_{1}, \ldots, \varphi_{*} V_{k}\right)=\mathfrak{D}\left(\nu \otimes \varphi^{*} \omega\right)\left(u, V_{1}, \ldots, V_{k}\right),
$$

for all $\boldsymbol{u} \in \mathbb{T}^{*}$ and $\boldsymbol{V}_{1}, \ldots, \boldsymbol{V}_{k} \in T \mathcal{R}_{0}$. Equation (13) provides a definition for $\mathfrak{D}$.

Useful identities. The following identities hold: ${ }^{5}$

$$
\left(\operatorname{div} \sigma^{\sharp 2}\right) \otimes \mu=\mathfrak{d}\left(*_{2} \sigma\right), \quad\left(\operatorname{Div} \mathbf{P}^{\sharp 2}\right) \otimes \mu_{0}=\mathfrak{D}\left(*_{2} \mathbf{P}\right) .
$$

where div and Div denote the divergence operator on $\mathcal{R}$ and $\mathcal{R}_{0}$, respectively,

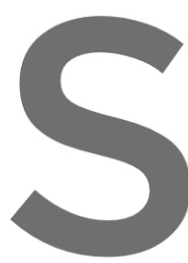

while $\sigma^{\sharp 2}=\sigma_{a}^{b} \mathrm{e}^{a} \otimes \boldsymbol{\phi}_{b}$ and $\mathbf{P}^{\mathrm{H}}=P_{A}^{B}$

'leg' only. Here, $\mu$ and $\mu_{0}$ are vollme forms in $\Omega^{3}(\mathcal{R})$

2.4. Balance laws

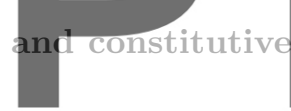

lations
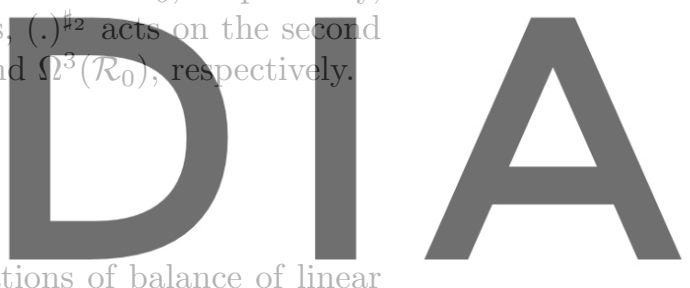

Balance of linear momemtum. The pointwise equations of balance of linear

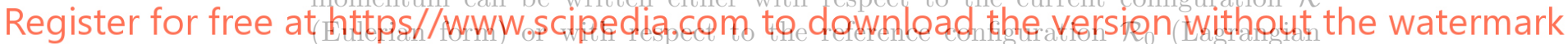
form) as follows

$$
\rho \dot{\mathbf{v}}^{b}=\operatorname{div} \boldsymbol{\sigma}^{\sharp 2}+\rho \mathbf{b}, \quad \rho_{0} \dot{\mathbf{V}}^{b}=\operatorname{Div} \mathbf{P}^{\sharp 2}+\rho_{0} \mathbf{B},
$$

where the overdot denotes the material time derivative. Here, $\rho$ is the mass density in $\mathcal{R}$, while $\rho_{0}$ is the referential mass density. Also, one has $\mathbf{B}(\mathbf{X}, t)=\mathbf{b}(\mathbf{x}(\mathbf{X}, t), t)$. It is worth emphasizing here that $\mathbf{V}(\mathbf{X}, t), \mathbf{B}(\mathbf{X}, t)$ and $\mathbf{p}(\mathbf{X}, t)$ are expressed in terms of a base point $\mathbf{X}$ in $\mathcal{R}_{0}$ but take their values in the tangent (or cotangent) fiber $T_{\mathbf{x}} \mathcal{R}$ (or $T_{\mathbf{x}}^{*} \mathcal{R}$ ) above the corresponding point $\mathbf{x}=\varphi(\mathbf{X}, t)$ in $\mathcal{R}$. Take the tensor product of the point-wise balance of linear momentum (15) with the volume forms $\mu$ and $\mu_{0}$, respectively, and use the identities in (14) to get

$$
\dot{\mathbf{v}}^{b} \otimes \rho \mu=\mathfrak{d} \mathcal{T}+\boldsymbol{b} \otimes \rho \mu, \quad \dot{\mathbf{V}}^{b} \otimes \rho_{0} \mu_{0}=\mathfrak{D} \mathcal{P}+\boldsymbol{B} \otimes \rho_{0} \mu_{0} .
$$

Balance of angular momemtum. Balance of angular momentum states that

$$
\boldsymbol{\sigma}^{\top}=\boldsymbol{\sigma}
$$

\footnotetext{
5 One can prove analogous results for any 2 -tensor.
} 
Consequently, the tensor $\mathbf{P F}^{\top}=J \boldsymbol{\sigma}$ is also symmetric. This symmetry translates in terms of $\mathcal{T}$, viewed as a vector-valued two-form, to the following equality:

$$
(\nu \otimes \beta) \dot{\wedge} \mathcal{T}=(\beta \otimes \nu) \dot{\wedge} \mathcal{T},
$$

for all $\nu, \beta \in \Omega^{1}(\mathcal{R})$. The symmetry of $\mathcal{T}$ can be interpreted physically as follows. Consider a surface $S_{\mathbf{n}}$ with unit normal $\mathbf{n}$ moving at a velocity $\mathbf{v}_{\mathbf{e}}=v \mathbf{e}$, where $\mathbf{e}$ is a unit vector. From (18), one gets that $\left(\mathbf{v}_{\mathbf{e}}^{b} \otimes \mathbf{n}^{b}\right) \dot{\wedge} \mathcal{T}=\mathbf{n}^{b} \wedge\left\langle\mathbf{v}_{\mathbf{e}}, \mathcal{T}\right\rangle=\mathbf{e}^{b} \wedge\left\langle\mathbf{v}_{\mathbf{n}}, \mathcal{T}\right\rangle$, where $\mathbf{v}_{\mathbf{n}}=v \mathbf{n}$. Combining this with (8), we see that the power per unit area expended by the stress as a surface $S_{\mathbf{n}}$ with unit normal $\mathbf{n}$ moves at a velocity $\mathbf{v}_{\mathrm{e}}=v \mathbf{e}$ is equal to the power per unit area expended by the stress as a surface $S_{\mathrm{e}}$ with unit normal e moves at a velocity $\mathrm{v}_{\mathrm{n}}=v \mathrm{n}$.

Finally, note that the statement of balance of angular momentum for $\mathcal{P}$ (as a vector-valued two-form) can be equivalently expressed as

$$
\left(\nu \otimes \varphi^{*} \beta\right) \dot{\wedge} \mathcal{P}=\left(\beta \otimes \varphi^{*} \nu\right) \dot{\wedge} \mathcal{P} \text {. }
$$

Conservation of mass. Recall that the pointwise equation of conservation of mass is usually written as
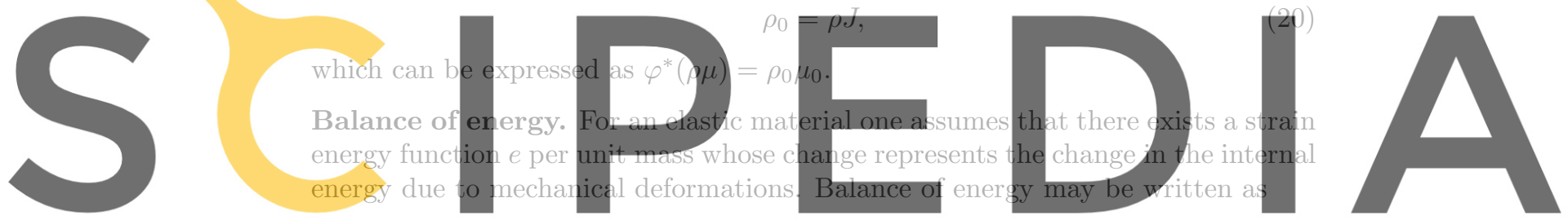

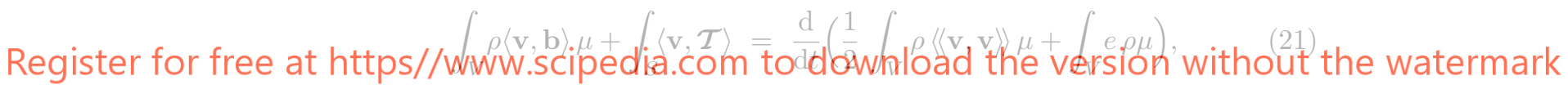
or, equivalently, on $\mathcal{R}_{0}$ as

$$
\int_{V_{0}} \rho_{0}\langle\mathbf{V}, \mathbf{B}\rangle \mu_{0}+\int_{S_{0}}\langle\mathbf{V}, \mathcal{P}\rangle=\frac{\mathrm{d}}{\mathrm{d} t}\left(\frac{1}{2} \int_{V_{0}} \rho_{0}\langle\langle\mathbf{V}, \mathbf{V}\rangle\rangle \mu_{0}+\int_{V_{0}} e \rho_{0} \mu_{0}\right) .
$$

Here, the $\langle\langle\rangle$,$\rangle denotes the inner products both on T \mathcal{R}$ and $T \mathcal{R}_{0}$, respectively. The volume integrals are taken over an arbitrary subset $V \subseteq \mathcal{R}$ while $V_{0}=\varphi^{-1}(V) \subseteq$ $\mathcal{R}_{0}$ and the area integrals are taken over the bounding surfaces $S=\partial V$ and $S_{0}=\partial V_{0}$. One can readily check, using Stokes' theorem, the definition of $\mathfrak{d}$ in $(10)$ and the balance of linear momentum (16), that the rate of change of internal energy is equal to

$$
\frac{\mathrm{d}}{\mathrm{d} t} \int_{V} e \rho \mu=\int_{V} \nabla \mathbf{v} \dot{\wedge} \mathcal{T}, \quad \frac{\mathrm{d}}{\mathrm{d} t} \int_{V_{0}} e \rho_{0} \mu_{0}=\int_{V_{0}} \nabla \mathbf{V} \dot{\wedge} \mathcal{P} .
$$

That is, the stress power can be expressed in terms of the stress form as in $\nabla \mathbf{v} \dot{\mathcal{T}}$ in $\mathcal{R}$ and $\nabla \mathbf{V} \dot{\wedge} \mathcal{P}$ in $\mathcal{R}_{0}$. In the classical theory, the stress power is defined as the inner product $\langle\langle\nabla \mathbf{v}, \boldsymbol{\sigma}\rangle\rangle$ in $\mathcal{R}$ or $\langle\langle\nabla \mathbf{V}, \mathbf{P}\rangle\rangle$ in $\mathcal{R}_{0}$, which can also be written as $\langle\langle\dot{\mathbf{F}}, \mathbf{P}\rangle\rangle$. 
We now present a simple counting argument. In general, $\mathcal{T}$ and $\mathcal{P}$ have nine independent components; balance of linear momentum (16) provides three independent equations of motion, and so does balance of angular momentum (18). Therefore, for a prescribed motion under given body forces, one has a system of nine unknowns and six equations. This means that to obtain a determinate system, one needs to impose constitutive relations on the stress $\mathcal{T}$ (or $\mathcal{P}$ ) as is done below for the case of hyperelastic materials.

Constitutive equations. The physical behavior of solid bodies depends on their material properties. Mathematically, a specific material, or class of materials, is characterized by a specific functional dependence of the stress tensor on the motion, that is, by a constitutive law. Hyperelastic materials have the property that the internal energy $e$ is function of $\mathbf{F}$ only, that is, $e=e(\mathbf{X}, \mathbf{F})$, and that the dependence of the stress on the motion is given by:

$$
\mathbf{P}^{\sharp 1}=\rho_{0} \frac{\partial e}{\partial \mathbf{F}}, \quad \sigma=2 \rho \frac{\partial e}{\partial \boldsymbol{g}}
$$

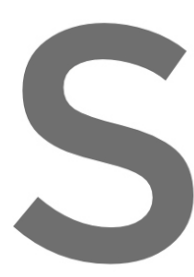

where the second part is known as the Doyle-Ericksen formula (here, $g=g^{i j} \mathbf{e}_{\text {, }}$
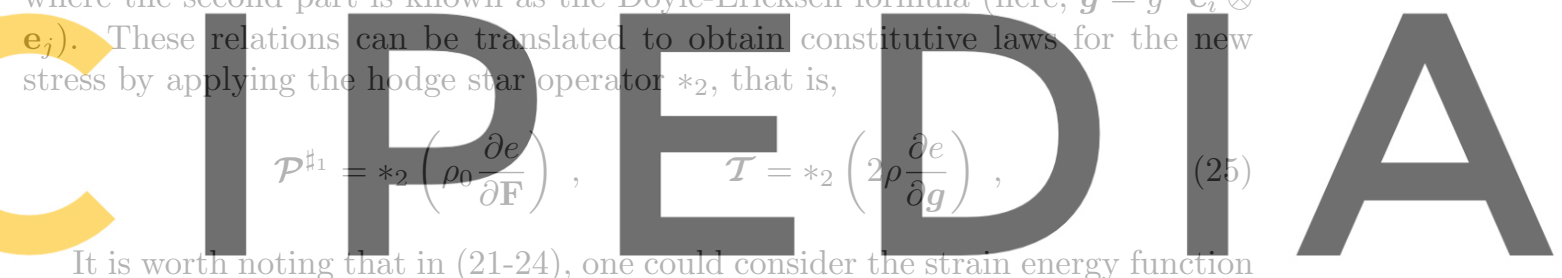

as an energy density or a volume form by treating ep $\mu$ as a single object, say

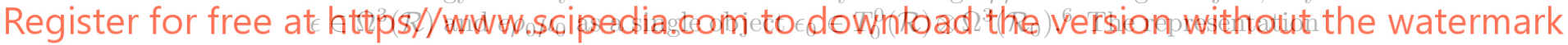

of the stress as a covector-valued two-form is consistent with the stress object one obtains from (24) using the energy density. For example, one can readily verify that $\mathcal{P}=\operatorname{trace}\left(\partial \epsilon_{0} / \partial \mathbf{F}\right)$.

Remark. The reader is reminded that the existence of the stress tensors (3) and the pointwise dynamical equations (15), (17) and (20) can be obtained by postulating integral laws of balance of momenta for the body, which is assumed to deform in the Euclidean space, or by postulating a covariant $t^{7}$ balance of energy for the body deforming in a general Riemannian manifold, see, [17] and [23]. It is important to note that the integral laws of balance of momenta are not intrinsic; the notion of a resultant force (or moment) explicitly utilizes the fact that the underlying physical space is Euclidean in a way that cannot be generalizable to curved Riemannian manifolds. In $\S 3$ we outline a procedure for deriving the pointwise balance laws from a fully geometric covariant theory of elasticity.

\footnotetext{
6 This notion of energy density is consistent with what is done in classical field theory, for example, in electromagnetism, the Lagrangian is not a scalar valued function but a density.

7 Here, by covariant we mean invariant under general coordinate transformations.
} 


\section{Covariant derivation of the balance laws}

In covariant elasticity, one starts from the balance of energy in (21) and postulates that it is invariant under arbitrary spatial diffeomorphisms $\xi_{t}: \mathcal{S} \rightarrow \mathcal{S}$, in order to derive the local forms of the conservation of mass, balance of momenta and the Doyle-Ericksen formula, see [17] and [23]. This covariant approach is a beautiful generalization of the classical Green-Rivlin-Naghdi results on invariance of energy balance under rotations and translations in Euclidean space. The main addition that one needs to make this covariant under general transformations are the stress constitutive relations.

In what follows, we assume the existence of a stress form $\mathcal{T}$, with no reference to the stress tensor $\boldsymbol{\sigma}$, and present a covariant derivation of the balance laws and constitutive equations. For notational convenience, define a scalar $f:=e+$ $\frac{1}{2}\langle\langle\mathbf{v}, \mathbf{v}\rangle\rangle ;$ one has

$$
\frac{d}{d t} \int_{V} f \rho \mu=\int_{V} \mathbf{L}_{\mathbf{v}}(f \rho \mu)=\int_{V} \rho \mu \mathbf{L}_{\mathbf{v}} f+f \mathbf{L}_{\mathbf{v}}(\rho \mu),
$$

where $\mathbf{L}_{\mathbf{v}}$ denotes the Lie derivative with respect to the velocity field $\mathbf{v}$ and $\mathbf{L}_{\mathbf{v}} f=$ $\dot{e}+\langle\langle\mathbf{v}, \dot{\mathbf{v}}\rangle\rangle$. Given a spatial diffeomorphism $\xi_{t}$, the balance of energy (21) can be rewritten in $\mathcal{S}$ as follows:

$$
\frac{d}{d t} \int_{V^{\prime}}\left(e^{\prime}+\frac{1}{2}\left\langle\left\langle\mathbf{v}^{\prime}, \mathbf{v}^{\prime}\right\rangle\right\rangle\right) \rho^{\prime} \mu^{\prime}=\int_{V^{\prime}} \rho^{\prime}\left\langle\mathbf{b}^{\prime}, \mathbf{v}^{\prime}\right\rangle \mu^{\prime}+\int_{S^{\prime}}\left\langle\mathcal{T}^{\prime}, \mathbf{v}^{\prime}\right\rangle .
$$

where the prime notation ()$^{\prime}$ is used to denote quantities in $\xi_{t}(\mathcal{S})$. In particular, one has $\rho \mu=\xi_{t}^{*}\left(\rho^{\prime} \mu^{\prime}\right)$ (conservation of mass) and $\mathbf{v}^{\prime}=\xi_{t *} \mathbf{v}+\mathbf{w}_{t}$, where $\mathbf{w}_{t}$ is the velocity of $\xi_{t}$. Further, one considers that the body force $\mathbf{b}$ transforms under spatial diffeomorphisms according to $\mathbf{b}-\dot{\mathbf{v}}^{b}=\xi_{t}^{*}\left(\mathbf{b}^{\prime}-\dot{\mathbf{v}}^{\prime} b\right)$, also, one has that the internal energy depends parametrically on the metric $e^{\prime}\left(\mathbf{x}^{\prime}, t, \mathbf{g}\right)=e\left(\mathbf{x}, t, \xi_{t}^{*} \mathbf{g}\right)$, (See [17, Chapter 2, Box 3.1].) Now, similarly to (26), one has

$$
\frac{d}{d t} \int_{V^{\prime}} f^{\prime} \rho^{\prime} \mu^{\prime}=\int_{V^{\prime}} \rho^{\prime} \mu^{\prime} \mathbf{L}_{\mathbf{v}^{\prime}} f^{\prime}+f^{\prime} \mathbf{L}_{\mathbf{v}^{\prime}}\left(\rho^{\prime} \mu^{\prime}\right)
$$

where $f^{\prime}=e^{\prime}+\frac{1}{2}\left\langle\left\langle\mathbf{v}^{\prime}, \mathbf{v}^{\prime}\right\rangle\right\rangle, \mathbf{L}_{\mathbf{v}^{\prime}}\left(\rho^{\prime} \mu^{\prime}\right)=\xi_{t *}\left(\mathbf{L}_{\mathbf{v}}(\rho \mu)\right)$ and $\mathbf{L}_{\mathbf{v}^{\prime}} f^{\prime}=\dot{e}^{\prime}+\left\langle\left\langle\mathbf{v}^{\prime}, \dot{\mathbf{v}}^{\prime}\right\rangle\right\rangle$. To this end, at $t=t_{0}$, one gets that $\left.f^{\prime}\right|_{t=t_{0}}=f+\langle\langle\mathbf{v}, \mathbf{w}\rangle\rangle+\frac{1}{2}\langle\langle\mathbf{w}, \mathbf{w}\rangle\rangle$, as well as $\left.\mathbf{L}_{\mathbf{v}^{\prime}}\left(\rho^{\prime} \mu^{\prime}\right)\right|_{t=t_{0}}=\mathbf{L}_{\mathbf{v}}(\rho \mu)$, and

$$
\left.\left(\mathbf{L}_{\mathbf{v}^{\prime}} f^{\prime}\right)\right|_{t=t_{0}}=\dot{e}+\left\langle\left\langle\frac{\partial e}{\partial \mathbf{g}}, \mathbf{L}_{\mathbf{w}} \mathbf{g}\right\rangle\right\rangle+\left\langle\left\langle\mathbf{v}+\mathbf{w}, \dot{\mathbf{v}}^{\prime}\right\rangle\right\rangle .
$$

Substitute (28-29) into (27) and subtract from (21) (in the resulting equation, the only term with $\langle\langle\mathbf{w}, \mathbf{w}\rangle\rangle$ is $\langle\langle\mathbf{w}, \mathbf{w}\rangle\rangle \mathbf{L}_{\mathbf{v}}(\rho \mu)$, hence, the arbitrariness of $\mathbf{w}$ leads to $\mathbf{L}_{\mathbf{v}}(\rho \mu)=0$, i.e., mass conservation). Take mass conservation into consideration and simplify the resulting equation to get

$$
\int_{V}\left\langle\left\langle\frac{\partial e}{\partial \mathbf{g}}, \mathbf{L}_{\mathbf{w}} \mathbf{g}\right\rangle\right\rangle \rho \mu=\int_{V}\left\langle\mathbf{w}, \mathbf{b}-\dot{\mathbf{v}}^{b}\right\rangle \rho \mu+\int_{S}\langle\mathbf{w}, \boldsymbol{T}\rangle .
$$


Apply Stokes' theorem to the last term and appeal to (10) to get

$$
\int_{V}\left\langle\left\langle\frac{\partial e}{\partial \mathbf{g}}, \mathbf{L}_{\mathbf{w}} \mathbf{g}\right\rangle\right\rangle \rho \mu=\int_{V}\left\langle\mathbf{w}, \mathbf{b}-\dot{\mathbf{v}}^{b}\right\rangle \rho \mu+\int_{V}\langle\mathbf{w}, \mathfrak{d} \mathcal{T}\rangle+\int_{V} \nabla \mathbf{w} \dot{\wedge} \mathcal{T} .
$$

Now $\nabla \mathbf{w}$ can be written as the sum of its symmetric and skew-symmetric parts, namely as

$$
(\nabla \mathbf{w})^{b}=\frac{1}{2} \mathbf{L}_{\mathbf{w}} \mathbf{g}+\mathrm{d}\left(\mathbf{w}^{b}\right)
$$

(it is important to note here that $\mathbf{g}$ is time independent). Because at any point, $\mathbf{w}, \mathbf{L}_{\mathbf{w}} \mathbf{g}$, and $\mathrm{d}\left(\mathbf{w}^{b}\right)$ can be chosen independently, one gets the balance of linear momentum (16), balance of angular momentum (18), and Doyle-Ericksen formula $(25)_{2}$.

\section{Summary}

This paper has presented a new and geometrically more natural formulation of continuum mechanics in terms of vector- and covector-valued forms, which are taken as replacements for (and are equivalent to) the standard stresses. It was shown that the formulation is equivalent to the classic theory by introducing mathematical operations on the relevant tangent and cotangent bundles. In this reformulation, the Cauchy stress field is replaced by a covector-valued two-form $\mathcal{T}$ which, when paired with the velocity field and integrated over a surface $S$, gives the rate of work done by the stress on that surface. Cauchy's Lemma is also automatically satisfied from the geometric nature of the stress $\mathcal{T}$. Finally, we presented a covariant derivation of the balance laws and constitutive relations in terms of $\mathcal{T}$ directly without the need to utilize the classical notion of stress as a two-tensor. As future work, it is planned to derive a discrete implementation of this geometric standpoint and compare it to recent numerical techniques $[12,7]$ that seem to share similar ideas and properties.

\section{References}

[1] Abraham, R., J.E. Marsden and T. Ratiu, Manifolds, Tensor Analysis, and Applications, Springer-Verlag, New York 1988.

[2] Cartan, E., On Manifolds with an Affine Connection and the Theory of General Relativity. Bibliopolis, Napoli 1995.

[3] Casey, J. and P. Papadopoulos, Material transport of sets and fields, Math. Mech. Sol. 7 (2002), 647-676.

[4] Desbrun, M., A. N. Hirani and J. E. Marsden, Discrete exterior calculus for variational problems in computer graphics, Proc. CDC, Hawaii 2003.

[5] Desbrun, M., E. Kanso and Y. Tong, Discrete differential forms for computational modeling, Chapter in "Discrete Differential Geometry" Course Notes, ACM SIGGRAPH 2005. 
[6] Di Carlo, A., A non-standard format for continuum mechanics, Contemporary Research in the Mechanics and Mathematics of Materials, R.C. Batra and M.F. Beatty, CIMNE, Barcelona 1996.

[7] Eyck, T. and Lew, A., Discontinuous Galerkin methods for nonlinear elasticity, IJNME 67 (2006), 1204-1243.

[8] Flanders, H., Differential Forms and Application to Physical Sciences, Dover 1990.

[9] Frankel, T., The Geometry of Physics, Cambridge University Press, United Kingdom 1997 (Second Edition 2004)

[10] Frölicher, A. and Nijenhuis, A., Theory of vector-valued differential forms. I. Derivations of the graded ring of differential forms, Indagationes Mathematics 18 (1956), 338-359.

[11] Green, A. E. and Rivilin, R. S., On Cauchy's equations of motion, ZAMP 15 (1964), 290-293.

[12] Hauret, P. and E. Kuhl, and M. Ortiz, Diamond elements: A finite-element/discretemechanics approximation scheme with guaranteed optimal convergence in incompressible elasticity, IJNME (2006), to appear.

[13] Hehl, F. W. and McCrea, J. D., Bianchi identities and the automatic conservation of energymomentum and angular momentum in general-relativistic field theories., Foundation of Physics 16 (1986), 267-293.

[14] Hirani, A. N., Discrete Exterior Calculus, Ph.D. thesis, California Institute of Technology 2003.

[15] Lewis, D., and J. C. Simo, Conservative Algorithms for the Dynamics of Hamiltonian Systems on Lie Groups, Nonlinear Science 4 (1994), 253.

[16] Madsen, I.H., and J. Tornehave, From Calculus to Cohomology : De Rham Cohomology and Characteristic Classes, Cambridge University Press 1997.

[17] Marsden, J. E. and T. J. R. Hughes, Mathematical Foundations of Elasticity, Dover, New York 1983.

[18] Segev R. and G. Rodnay, Cauchy's theorem on manifolds, Journal of Elasticity 56 (1999), 129-144.

[19] Simo, J. C. and N. Tarnow, The discrete energy momentum method:conserving algorithms for nonlinear elastodynamics, Zeitschrift fr Angewandte Mathematik und Physik (ZAMP) 43 (1992), 757-792.

[20] Simo, J. C., N. Tarnow and K. K. Wong, Exact energy-momentum conserving algorithms and symplectic schemes for nonlinear dynamics, Computer Methods in Applied Mechanics and Engineering 100 (1992), 63-116.

[21] Vargas, J. .G. and D. G. Torr, Conservation of vector-valued forms and the question of the existence of gravitational energy-momentum in general relativity. General Relativity and Gravitation, 23 (1991), 713-732.

[22] West, M. and J. E. Marsden, Discrete mechanics and variational integrators, Acta Numerica (2001), 357-514.

[23] Yavari, A., J. E. Marsden and M. Ortiz, On the spatial and material covariant balance laws in elasticity, Journal of Mathematical Physics 47 (2006), 1-53.

Eva Kanso

University of Southern California

Los Angeles, CA 90089

USA

Marino Arroyo

Universitat Politècnica de Catalunya

Barcelona

Spain 
E. Kanso et al.

Yiying Tong

California Institute of Technology

Pasadena, CA 91125

Arash Yavari

Georgia Institute of Technology

Atlanta, GA 30332

USA

Mathieu Desbrun

California Institute of Technology

Pasadena, CA 91125

USA 